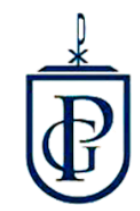

Veritas Et Scientia

Vol. $7, N^{\circ} 1,848-858$

Enero - Junio del 2018.

ISSN $2307-5139$

\title{
ISOVALORES DE CAPACIDAD ADMISIBLE DEL SUELO PARA CIMENTACIONES SUPERFICIALES EN EL SECTOR SIETE DEL DISTRITO ALTO DE LA ALIANZA, TACNA 2017.
}

ISOVALUES OF ADMISSIBLE SOIL CAPACITY FOR SUPERFICIAL FOUNDATIONS IN THE SEVEN SECTOR OF THE HIGH DISTRICT OF ALTO DE LA ALIANZA, TACNA 2017.

Pedro Valerio Maquera $\mathrm{Cruz}^{1}$

\section{RESUMEN}

La presente tesis analiza el problema del uso de suelos para cimentaciones de viviendas en áreas o terrenos no habilitados o posesionados por invasiones. El objetivo es caracterizar la capacidad admisible del suelo para cimentaciones superficiales del sector siete del distrito de Alto de la Alianza, Provincia y Región de Tacna. 2017. Investigación prospectiva, la población está referida a los suelos de la zona y la muestra corresponde a las calicatas estudiadas. Los resultados de los suelos de las exploraciones realizadas indican que las propiedades físicas son similares, siendo el suelo una arena limosa no plástica. La cohesión se ha considerado cero por ser un suelo arenoso limoso, y el ángulo de fricción en promedio de $30^{\circ}$ en estado natural. El valor de la capacidad admisible de carga ha sido calculado para cimentaciones superficiales a distintas profundidades de desplante y para diferentes anchos de cimentación. Se concluye una caracterización de isovalores de la capacidad admisible del suelo que están por debajo de $1 \mathrm{~kg} / \mathrm{cm} 2$ para una profundidad de desplante de $0.80 \mathrm{~m}$ y $1.20 \mathrm{~m}$. con un ancho de la cimentación de 1.0 $\times 1.0 \mathrm{~m}$.

Palabras clave: Calicatas, parámetros de resistencia, capacidad admisible.

\footnotetext{
${ }^{1}$ Magíster en ingeniería civil con mención en geotecnia
} 


\begin{abstract}
This thesis analyzes the problem of the use of soils for foundations of houses in areas or lands not enabled or possessed by invasions. The objective is to characterize the admissible capacity of the ground for superficial foundations of sector seven of the district of "Alto de la Alianza", Province and Region of Tacna. 2017. Prospective research, the population is referred to the soils of the area and the sample corresponds to the pits studied. The results of the soils of the explorations carried out indicate that the physical properties are similar, the soil being a non-plastic silty sand. The cohesion has been considered zero for being a sandy loamy soil, and the angle of friction in average of $30^{\circ}$ in natural state. The value of the admissible load capacity has been calculated for surface foundations at different depths of slab and for different foundation widths. We conclude a characterization of isovalues of the admissible soil capacity that are below $1 \mathrm{~kg} / \mathrm{cm} 2$ for a depth of $0.80 \mathrm{~m}$ and $1.20 \mathrm{~m}$. with a foundation width of $1.0 \times 1.0 \mathrm{~m}$.
\end{abstract}

Keywords: Calicates, resistance parameters, admissible capacity.

\title{
INTRODUCCIÓN
}

El crecimiento demográfico de la ciudad de Tacna ha propiciado que muchas familias migrantes, se asienten en zonas no aptas en resistencia para la cimentación de viviendas de diversas regiones del país. Familias provenientes de Puno, Moquegua, Cuzco, Arequipa y Norte del país, se han asentado en el cono Norte de la Ciudad de Tacna; específicamente la parte alta de la ciudad colindante con la Carretera Tacna - Tarata entre los Km. 6 al km. 7. A este sector se le conoce como el Sector 7 del Distrito Alto de la Alianza. Según el PDU 2015-2025, Plan de Desarrollo Urbano de Tacna, en la zona se han formado varias Asociaciones de Vivienda como Taller, Pequeña Industria, Pecuario, entre otros. Los análisis de capacidad portante realizados a los suelos, con fines de cimentaciones de viviendas, es muy escaso o con varios años de antigüedad. Se cuenta con estudios de proyectos para Ciudades Sostenibles realizados por $I N D E C l$, que indican que la zona presenta materiales de baja capacidad portante y recomendando su uso para actividades de industria, comercio, vivienda taller. El Plan de Desarrollo Urbano - 2015 al 2025, aprobado por la Municipalidad de Tacna, plantea usos de suelos para pequeña Industria (vivienda taller), pecuario y otros. Sin embargo la mayoría de los posesionarios, construyen sus viviendas de manera irresponsable, alcanzando edificaciones de hasta dos niveles en la zona de estudio. El uso de suelos para viviendas por medio de las invasiones, ha generado que muchas familias se asienten sobre suelos sin algún estudio previo que garantice la sostenibilidad de las viviendas a edificar. La ciudad de Tacna sigue creciendo sin planificación y sin ordenamiento urbano, especialmente aquellos lugares que deben ser definidos por los análisis de suelos o por la capacidad de carga admisible. Gran parte del cono norte presenta suelos de baja capacidad portante, donde se recomienda no sean habitados, sin embargo, en dichas zonas los pobladores siguen construyendo sus viviendas. En la Asociación de Vivienda Taller Héroes Alto de la Alianza, el $100 \%$ de las construcciones corresponden a material noble (cercos perimétricos, viviendas, galpones, etc.). Por otro lado las asociaciones dedicadas a la actividad pecuaria presentan construcciones de material noble, en manzanas o lotes ubicadas cercas a la carretera Tacna - Tarata ( $\mathrm{km} \mathrm{6)}$. Estos lotes están cambiando de uso, de actividad pecuaria a las actividades comercial de ferretería, tiendas dedicadas a la venta de productos para animales, locales dedicados a la 
actividad de restaurantes, cocheras, pequeños talleres, entre otras actividades. El resto de lotes de las otras asociaciones están dedicados a la actividad pecuaria. Los terrenos se encuentran cercados a base de palos y mallas de pescador, con pocas y pequeñas construcciones dentro de algunos lotes.

El Proyecto INDECI - PNUD PER/02/051(2004), para ciudades sostenibles - Tacna desarrolló mapas de peligros de la ciudad de Tacna, para los distritos de Tacna, Gregorio Albarracín, Pocollay y Complemento Alto de la Alianza y Ciudad Nueva. En su resumen ejecutivo se indica que los distritos de Alto de la Alianza y Ciudad Nueva, en sus actuales áreas urbanas, los suelos presentan malas características geotécnicas, con amplificación sísmica elevada y capacidades portantes menores a 1.0 $\mathrm{kg} / \mathrm{cm}^{2}$. El Instituto Nacional de Defensa Civil - INDECl, ejecutó, a través del Proyecto PNUD PER/02/051 00014426, el Programa Ciudades Sostenibles. El estudio hace mención a que los suelos localizados al Nor este (alto de la Alianza y Ciudad Nueva), en contraposición; presentan condiciones inadecuadas para soportar edificaciones.

Terzaghi fue el primero en presentar una teoría completa para evaluar la capacidad de carga última de cimentaciones superficiales. De acuerdo con esta, una cimentación es superficial si la profundidad Df de la cimentación es menor o igual que el ancho de la misma.

\section{OBJETIVOS}

- Realizar ensayos básicos y ensayos de resistencia al esfuerzo cortante, para caracterizar los suelos por las propiedades físicas y de resistencia de los suelos areno limosos.

- Determinar valores de la capacidad admisible de los suelos, que permitan la construcción de mapas temáticos de isovalores.

- Elaborar mapas de isovalores de capacidades de carga,para que las autoridades tengan una herramienta para la toma de decisiones.

\section{METODOLOGÍA}

La investigación es del tipo aplicada, propositiva. La población objeto del estudio, está compuesto por 08 calicatas distribuidas de manera equidistantes en la zona de investigación. El estudio determinará valores de capacidad de carga admisibles para distintas profundidades y dimensiones de cimentación superficial de forma cuadrada. Las técnicas se basan en la realización de ensayos, diseños y planos, que consideran las recomendaciones de las normas y reglamentos. Los equipos básicos determinaron los ensayos básicos siguientes:

- $\quad$ Contenido de Humedad NTP. 339.127 (ASTM D2216)

- $\quad$ Densidad Insitu NTP 339.143

- $\quad$ Análisis Granulométrico x tamizado NTP 339.128 (ASTM D422)

- $\quad$ Límite líquido y Límite plástico NTP 339.129 (ASTM D4318)

- Clasificación Unificada de Suelos SUCS NTP 339.134 (ASTM D2487)

Se contó con equipo de corte con capacidad de producir esfuerzos normales y horizontales de corte; dotado con un carro móvil y caja donde se colocará la muestra entre piedras porosas para permitir el drenaje. Deformímetros para registrar asentamientos y deformaciones horizontales. Además, una celda que registre con precisión las fuerzas de corte aplicadas. El área de estudio se ubica en el Sector siete en la planicie del Alto Tacna del Distrito Alto de la Alianza Provincia y Región de Tacna. Colindante a la Carretera Tacna Tarata, entre los km 6+000 y km 7+000. 


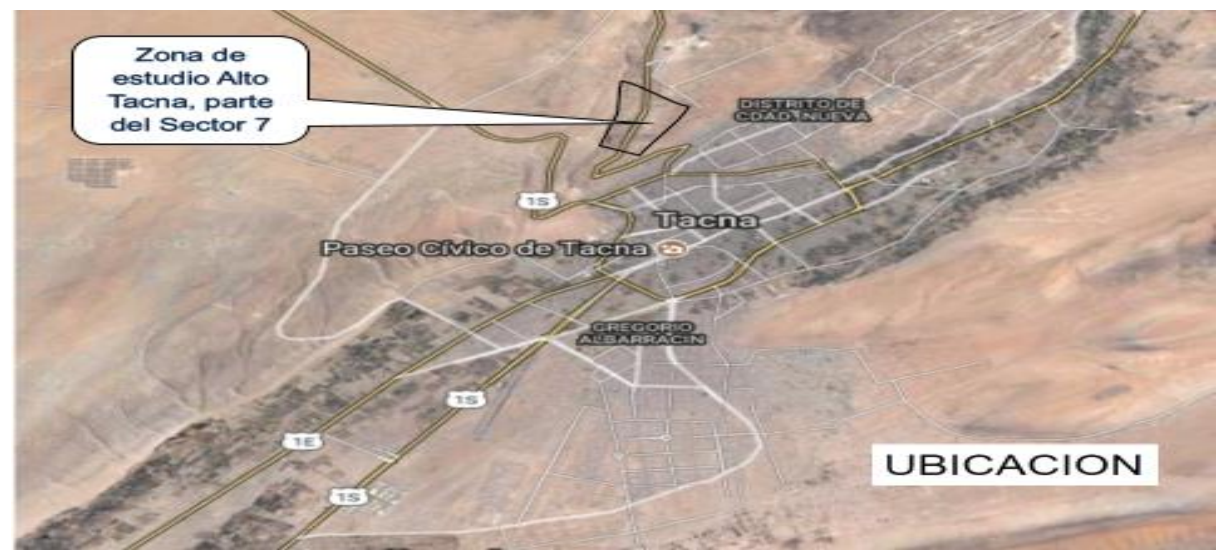

Figura 1. Mapa de ubicación de zona de investigación

El área de estudio Alto Tacna (sector siete) presenta una topografía que varía de plano a ondulado. El terreno presenta pendientes variables ascendente de Oeste a Este entre $3.0 \%$ a $4.6 \%$. De acuerdo al nuevo mapa de zonificación sísmica del Perú, según la nueva Norma Sismo Resistente (NTE-030); se concluye que el área en estudio se encuentra dentro de la zona de Sismicidad (Zona 3).

Una vez realizado las calicatas se ha procedido a realizar el ensayo de densidad in situ, luego se ha tomado muestras alteradas para realizar los ensayos en el Laboratorio:

Los ensayos básicos y especiales realizados fueron:

- Contenido de humedad NTP. 339.127 (ASTM D2216)

- Densidad in situ NTP 339.143

- Análisis granulométrico por tamizado NTP 339.128 (ASTM D422)

- Límite líquido y Límite plástico NTP 339.129 (ASTM D4318)

- Clasificación Unificada de Suelos SUCS NTP 339.134 (ASTM D2487)

- Ensayo de Corte Directo ASTM D3080

- $\quad$ Ensayo de Penetración DPL ASTM D 3441 NTP 339.159

Resumen de Ensayos básicos

\begin{tabular}{|c|c|c|c|c|c|c|c|c|c|}
\hline \multirow[t]{2}{*}{ Calicata } & \multirow{2}{*}{ Ubicación } & \multicolumn{2}{|c|}{$\begin{array}{l}\text { Densidad de } \\
\text { campo }\end{array}$} & \multirow{2}{*}{$\begin{array}{l}\text { Prof } \\
(\mathrm{m})\end{array}$} & \multicolumn{3}{|c|}{ Plasticidad } & \multicolumn{2}{|c|}{ Granulometría } \\
\hline & & DMS & $\mathrm{H}(\%)$ & & $\mathrm{LL}$ & $\mathrm{LP}$ & IP & Pas\#4 & Pas\#200 \\
\hline C-1 & $\begin{array}{l}\text { Asoc. criadores de animales } \\
\text { menores Alto Tacna }\end{array}$ & 1.58 & 1.30 & 2.50 & 18 & NP & NP & 99.2 & 19.0 \\
\hline C-2 & $\begin{array}{l}\text { Asoc. criadores de porcinos } \\
\text { Virgen de Chapí }\end{array}$ & 1.79 & 2.1 & 0.80 & 16.5 & NP & NP & 100 & 18.9 \\
\hline$C-3$ & $\begin{array}{l}\text { Asoc. criadores de animales } \\
\text { menores Las Pascuas }\end{array}$ & 1.61 & 1.46 & 1.10 & 19 & NP & NP & 93.1 & 14.1 \\
\hline C-4 & $\begin{array}{l}\text { Asoc. de granjas Nueva } \\
\text { Tarata }\end{array}$ & 1.71 & 1.53 & 2.00 & 17.1 & NP & NP & 100 & 14.5 \\
\hline$C-5$ & $\begin{array}{l}\text { Asoc. Artesanal Taller } \\
\text { Vivienda Héroes del Alto de } \\
\text { la Alianza }\end{array}$ & 1.55 & 1.68 & 1.20 & 16.3 & NP & NP & 100 & 12.4 \\
\hline C-6 & $\begin{array}{l}\text { Asoc. Hermandad de la } \\
\text { Stma. Cruz del cerro Intiorko }\end{array}$ & 1.60 & 2.0 & 2.20 & 17.2 & NP & NP & 98.1 & 20.3 \\
\hline$C-7$ & $\begin{array}{l}\text { Asoc. criadores de aves Alto } \\
\text { Santa Cruz Cerro Intiorko }\end{array}$ & 1.87 & 1.21 & 0.90 & 17.7 & NP & NP & 95.9 & 16.7 \\
\hline C.8 & $\begin{array}{l}\text { Asoc. criadores de animales } \\
\text { menores Las Vegas }\end{array}$ & 1.7 & 1.4 & 0.80 & 15.5 & NP & NP & 100 & 12.1 \\
\hline
\end{tabular}


Las determinaciones de los parámetros de resistencia se han realizado mediante ensayos de DPL (in situ) y ensayos de corte directo (en laboratorio), que de acuerdo a su proceso se obtienen cohesión y ángulo de fricción del suelo.

El ensayo DPL se realizó en las calicatas, C1, C5 y C7. Este ensayo corresponde al hincado continuo en tramos de $10 \mathrm{~cm}$ de una punta cónica de 60 utilizando la energía de un martillo de $10 \mathrm{~kg}$ de peso, que cae libremente desde una altura de $50 \mathrm{~cm}$. Este ensayo proporciona el registro continuo de resistencia del terreno a la penetración, existiendo correlaciones para encontrar el valor " $\mathrm{N}$ " de resistencia a la penetración estándar en función del tipo de suelo, para cada $30 \mathrm{~cm}$ de hincado.

La cohesión se ha considerado como nula, dado que el suelo se clasifica como un suelo arenoso limoso. El ángulo de fricción mínimo obtenido para la muestra de la calicata C-8 fue de $25^{\circ}$ y el máximo de $31.51^{\circ}$ para la muestra de la calicata C-1.

Resumen de ensayos especiales de DPL y Corte Directo

\begin{tabular}{|l|c|c|c|c|c|}
\hline$z(m)$ & $\mathbf{m}=\mathbf{n}$ & $\mathbf{I}_{\mathbf{o}}$ & $\mathbf{q}\left(\mathbf{T n} / \mathbf{m}^{2}\right)$ & $\sigma_{\mathbf{z}}\left(\mathbf{T n} / \mathbf{m}^{2}\right)$ & $\sigma_{\mathbf{z}}\left(\mathbf{x} \mathbf{(}\left(\mathbf{T n} / \mathbf{m}^{2}\right)\right)$ \\
\hline $\mathbf{0 . 0 0}$ & - & - & 5 & 5 & $\mathbf{5 . 0 0}$ \\
\hline $\mathbf{0 . 5 0}$ & 2 & 0.233 & 5 & 1.165 & 4.66 \\
\hline $\mathbf{1 . 0 0}$ & 1 & 0.17 & 5 & 0.85 & 3.40 \\
\hline $\mathbf{1 . 5 0}$ & 0.667 & 0.125 & 5 & 0.625 & 2.50 \\
\hline $\mathbf{2 . 0 0}$ & 0.5 & 0.075 & 5 & 0.375 & 1.50 \\
\hline $\mathbf{2 . 5 0}$ & 0.4 & 0.058 & 5 & 0.29 & 1.16 \\
\hline $\mathbf{3 . 0 0}$ & 0.333 & 0.045 & 5 & 0.225 & 0.90 \\
\hline $\mathbf{3 . 5 0}$ & 0.286 & 0.03 & 5 & 0.15 & 0.60 \\
\hline $\mathbf{4 . 0 0}$ & 0.25 & 0.02 & 5 & 0.10 & 0.40 \\
\hline
\end{tabular}

Los valores de la capacidad admisible de los suelos del área de estudio, se determinan en base al marco teórico, con la información de campo y laboratorio.

Los resultados obtenidos de los esfuerzos verticales a profundidades que van variando cada $1 / 2$ metro hasta una profundidad de $4 \mathrm{~m}$. Se muestran en la tabla siguiente.

Esfuerzo vertical centro de la zapata vs desplazamiento vertical

\begin{tabular}{|c|c|c|c|c|c|c|c|}
\hline \multirow{2}{*}{ Calicata } & \multirow[t]{2}{*}{ Ubicación } & \multicolumn{2}{|c|}{$\begin{array}{l}\text { Corte } \\
\text { Directo }\end{array}$} & \multirow[t]{2}{*}{ SUCS } & \multicolumn{3}{|c|}{$\mathrm{DPL}(\mathrm{c} / 10 \mathrm{~cm})$} \\
\hline & & C & $0\left(^{\circ}\right)$ & & 50 & 100 & 250 \\
\hline$C-1$ & $\begin{array}{l}\text { Asoc. criadores de animales } \\
\text { menores Alto Tacna }\end{array}$ & 0.00 & 31.5 & SM & 8 & 8 & 49 \\
\hline$C-2$ & $\begin{array}{l}\text { Asoc. criadores de porcinos } \\
\text { Virgen de Chapí }\end{array}$ & 0.00 & 30.3 & SM & & & \\
\hline$C-3$ & $\begin{array}{l}\text { Asoc. criadores de animales } \\
\text { menores Las Pascuas }\end{array}$ & 0.00 & 26.5 & SM & & & \\
\hline C-4 & Asoc. de granjas Nueva Tarata & 0.00 & 28.5 & SM & & & \\
\hline$C-5$ & $\begin{array}{l}\text { Asoc. Artesanal Taller Vivienda } \\
\text { Héroes del Alto de la Alianza }\end{array}$ & 0.00 & 29.3 & SM & 11 & 48 & \\
\hline$C-6$ & $\begin{array}{l}\text { Asoc. Hermandad de la Santísima } \\
\text { Cruz del cerro Intiorko }\end{array}$ & 0.00 & 29.5 & SM & & & \\
\hline C-7 & $\begin{array}{l}\text { Asoc. criadores de aves Alto } \\
\text { Santa Cruz Cerro intiorko }\end{array}$ & 0.00 & 28.5 & SM & 10 & 47 & \\
\hline C.8 & $\begin{array}{l}\text { Asoc. criadores de animales } \\
\text { menores Las Vegas }\end{array}$ & 0.00 & 25.2 & SM & & & \\
\hline
\end{tabular}


Para determinar la capacidad portante del suelo se ha utilizado la expresión de Terzaghi

Aplicando las ecuaciones de Terzaghi se obtiene una carga última para zapatas cuadradas de:

qult $=1.3 * 0 * 15+1.58 * 1.2 * 10.2+0.4 * 1.0 * 1.58 * 8.2$

qult $=24.5 \mathrm{tn} / \mathrm{m}^{2}$.

Utilizando $\mathrm{FS}=3$, la Capacidad admisible de los suelos es: $\mathbf{q a d m}=\mathbf{0 . 8 1} \mathbf{~} \mathbf{g} / \mathrm{cm}^{2}$. Valor para cimientos cuadrados

\section{RESULTADOS}

Las construcciones de las edificaciones están siendo cimentadas sin los estudios de suelos correspondientes y sin las licencias de edificación. Se observan viviendas de 2 a 3 niveles, las cuales se encuentran mayormente colindantes con la carretera Tacna - Tarata ( $\mathrm{Km} \mathrm{6)}$. Existe demanda por la utilización de los suelos para edificaciones con cimentaciones superficiales para fines de vivienda y comercio. La capacidad admisible de los suelos, consideró un factor de seguridad de 3 . Con el suelo rocoso y elevada compacidad, se consideró falla general. Para un ancho (B) de la cimentación corrida y profundidad de desplante (Df), se consideró dimensiones para zapatas cuadradas de $B=0.80 ; 1.00 ; 1.20$; 1.40 y $1.60 \mathrm{~m}$. Para diferentes profundidades de desplante de una zapatas aisladas (cuadrada) se comparó los resultados de las diferentes calicatas y se obtuvo valores característicos de la capacidad portante del suelo. Se analizó la profundidad de desplante, según el perfil estratigráfico, entre $0.8 \mathrm{~m}$ a $2.50 \mathrm{~m}$.

Tabla 1. Valores de Capacidad admisible para la Calicata C-1

\begin{tabular}{|c|c|c|c|c|c|c|c|}
\hline Df $(m)$ & $B(m)$ & $\begin{array}{l}\text { Q(ult) } \\
\text { Tn/m2 }\end{array}$ & \begin{tabular}{|c|}
$\mathrm{Q}(\mathrm{adm})$ \\
$\mathrm{Kg} . / \mathrm{cm} 2$ \\
\end{tabular} & Df $(m)$ & $B(m)$ & $\begin{array}{l}\text { Q(ult) } \\
\text { Tn/m2 }\end{array}$ & \begin{tabular}{|l} 
Q(adm) \\
$\mathrm{Kg} . / \mathrm{cm} 2$ \\
\end{tabular} \\
\hline 0.60 & 0.80 & 13.78 & 0.46 & 1.50 & 0.80 & 28.23 & 0.94 \\
\hline 0.60 & 1.00 & 14.81 & 0.49 & 1.50 & 1.00 & 29.27 & 0.98 \\
\hline 0.60 & 1.20 & 15.84 & 0.53 & 1.50 & 1.20 & 30.30 & 1.01 \\
\hline 0.60 & 1.40 & 16.88 & 0.56 & 1.50 & 1.40 & 31.34 & 1.04 \\
\hline 0.60 & 1.60 & 17.91 & 0.60 & 1.50 & 1.60 & 32.37 & 1.08 \\
\hline 0.80 & 0.80 & 16.99 & 0.57 & 2.00 & 0.80 & 36.26 & 1.21 \\
\hline 0.80 & 1.00 & 18.02 & 0.60 & 2.00 & 1.00 & 37.30 & 1.24 \\
\hline 0.80 & 1.20 & 19.06 & 0.64 & 2.00 & 1.20 & 38.33 & 1.28 \\
\hline 0.80 & 1.40 & 20.09 & 0.67 & 2.00 & 1.40 & 39.37 & 1.31 \\
\hline 0.80 & 1.60 & 21.13 & 0.70 & 2.00 & 1.60 & 40.40 & 1.35 \\
\hline 1.00 & 0.80 & 20.20 & 0.67 & 2.50 & 0.80 & 44.29 & 1.48 \\
\hline 1.00 & 1.00 & 21.23 & 0.71 & 2.50 & 1.00 & 45.33 & 1.51 \\
\hline 1.00 & 1.20 & 22.27 & 0.74 & 2.50 & 1.20 & 46.36 & 1.55 \\
\hline 1.00 & 1.40 & 23.30 & 0.78 & 2.50 & 1.40 & 47.40 & 1.58 \\
\hline 1.00 & 1.60 & 24.34 & 0.81 & 2.50 & 1.60 & 48.43 & 1.61 \\
\hline 1.20 & 0.80 & 23.41 & 0.78 & 3.00 & 0.80 & 52.33 & 1.74 \\
\hline 1.20 & 1.00 & 24.45 & 0.81 & 3.00 & 1.00 & 53.36 & 1.78 \\
\hline 1.20 & 1.20 & 25.48 & 0.85 & 3.00 & 1.20 & 54.40 & 1.81 \\
\hline 1.20 & 1.40 & 26.52 & 0.88 & 3.00 & 1.40 & 55.43 & 1.85 \\
\hline 1.20 & 1.60 & 27.55 & 0.92 & 3.00 & 1.60 & 56.46 & 1.88 \\
\hline
\end{tabular}


Tabla 2. Valores de Capacidad admisible para la Calicata C-2

\begin{tabular}{|c|c|c|c|}
\hline Df $(\mathbf{m})$ & $\mathbf{B}(\mathbf{m})$ & $\begin{array}{c}\mathbf{Q}(\mathbf{u l t}) \\
\mathbf{T n} / \mathbf{m} 2\end{array}$ & $\begin{array}{c}\mathbf{Q}(\mathbf{a d m}) \\
\text { Kg./cm2 }\end{array}$ \\
\hline 0.60 & 0.80 & 14.06 & 0.47 \\
\hline 0.60 & 1.00 & 15.09 & 0.50 \\
\hline 0.60 & 1.20 & 16.12 & 0.54 \\
\hline 0.60 & 1.40 & 17.15 & 0.57 \\
\hline 0.60 & 1.60 & 18.18 & 0.61 \\
\hline 0.80 & 0.80 & 17.37 & 0.58 \\
\hline 0.80 & 1.00 & 18.40 & 0.61 \\
\hline 0.80 & 1.20 & 19.43 & 0.65 \\
\hline 0.80 & 1.40 & 20.46 & 0.68 \\
\hline 0.80 & 1.60 & 21.49 & 0.72 \\
\hline 1.00 & 0.80 & 20.68 & 0.69 \\
\hline 1.00 & 1.00 & 21.71 & 0.72 \\
\hline 1.00 & 1.20 & 22.74 & 0.76 \\
\hline 1.00 & 1.40 & 23.77 & 0.79 \\
\hline 1.00 & 1.60 & 24.80 & 0.83 \\
\hline
\end{tabular}

Tabla 3. Valores de Capacidad admisible para la Calicata C-3

\begin{tabular}{|c|c|c|c|}
\hline Df $(m)$ & $B(m)$ & $\begin{array}{c}\text { Q(ult) } \\
\text { Tn/m2 }\end{array}$ & $\begin{array}{c}\text { Q(adm) } \\
\text { Kg./cm2 }\end{array}$ \\
\hline 0.60 & 0.80 & 9.01 & 0.30 \\
\hline 0.60 & 1.00 & 9.62 & 0.32 \\
\hline 0.60 & 1.20 & 10.22 & 0.34 \\
\hline 0.60 & 1.40 & 10.83 & 0.36 \\
\hline 0.60 & 1.60 & 11.43 & 0.38 \\
\hline 0.80 & 0.80 & 11.21 & 0.37 \\
\hline 0.80 & 1.00 & 11.81 & 0.39 \\
\hline 0.80 & 1.20 & 12.42 & 0.41 \\
\hline 0.80 & 1.40 & 13.02 & 0.43 \\
\hline 0.80 & 1.60 & 13.63 & 0.45 \\
\hline 1.00 & 0.80 & 13.40 & 0.45 \\
\hline 1.00 & 1.00 & 14.01 & 0.47 \\
\hline 1.00 & 1.20 & 14.61 & 0.49 \\
\hline 1.00 & 1.40 & 15.22 & 0.51 \\
\hline 1.00 & 1.60 & 15.82 & 0.53 \\
\hline 1.20 & 0.80 & 15.60 & 0.52 \\
\hline 1.20 & 1.00 & 16.20 & 0.54 \\
\hline 1.20 & 1.20 & 16.81 & 0.56 \\
\hline 1.20 & 1.40 & 17.41 & 0.58 \\
\hline 1.20 & 1.60 & 18.02 & 0.60 \\
\hline
\end{tabular}

Tabla 4. Valores de Capacidad admisible para la Calicata C-4

\begin{tabular}{|c|c|c|c|c|c|c|c|}
\hline Df $(m)$ & $B(m)$ & $\begin{array}{l}\text { Q(ult) } \\
\text { Tn/m2 }\end{array}$ & $\begin{array}{l}\mathrm{Q}(\mathrm{adm}) \\
\mathrm{Kg} . / \mathrm{cm} 2 \\
\end{array}$ & Df $(m)$ & $B(m)$ & $\begin{array}{l}\text { Q(ult) } \\
\text { Tn/m2 }\end{array}$ & $\begin{array}{c}\text { Q(adm) } \\
\mathrm{Kg} . / \mathrm{cm} 2\end{array}$ \\
\hline 0.60 & 0.80 & 11.40 & 0.38 & 1.50 & 0.80 & 23.68 & 0.79 \\
\hline 0.60 & 1.00 & 12.20 & 0.41 & 1.50 & 1.00 & 24.49 & 0.82 \\
\hline 0.60 & 1.20 & 13.01 & 0.43 & 1.50 & 1.20 & 25.29 & 0.84 \\
\hline 0.60 & 1.40 & 13.81 & 0.46 & 1.50 & 1.40 & 26.09 & 0.87 \\
\hline 0.60 & 1.60 & 14.61 & 0.49 & 1.50 & 1.60 & 26.90 & 0.90 \\
\hline 0.80 & 0.80 & 14.13 & 0.47 & 2.00 & 0.80 & 30.51 & 1.02 \\
\hline 0.80 & 1.00 & 14.93 & 0.50 & 2.00 & 1.00 & 31.31 & 1.04 \\
\hline 0.80 & 1.20 & 15.74 & 0.52 & 2.00 & 1.20 & 32.11 & 1.07 \\
\hline 0.80 & 1.40 & 16.54 & 0.55 & 2.00 & 1.40 & 32.92 & 1.10 \\
\hline 0.80 & 1.60 & 17.34 & 0.58 & 2.00 & 1.60 & 33.72 & 1.12 \\
\hline 1.00 & 0.80 & 16.86 & 0.56 & 2.50 & 0.80 & 37.33 & 1.24 \\
\hline 1.00 & 1.00 & 17.66 & 0.59 & 2.50 & 1.00 & 38.14 & 1.27 \\
\hline 1.00 & 1.20 & 18.47 & 0.62 & 2.50 & 1.20 & 38.94 & 1.30 \\
\hline 1.00 & 1.40 & 19.27 & 0.64 & 2.50 & 1.40 & 39.74 & 1.32 \\
\hline 1.00 & 1.60 & 20.07 & 0.67 & 2.50 & 1.60 & 40.55 & 1.35 \\
\hline 1.20 & 0.80 & 19.59 & 0.65 & & & & \\
\hline 1.20 & 1.00 & 20.39 & 0.68 & & & & \\
\hline 1.20 & 1.20 & 21.20 & 0.71 & & & & \\
\hline 1.20 & 1.40 & 22.00 & 0.73 & & & & \\
\hline 1.20 & 1.60 & 22.80 & 0.76 & & & & \\
\hline
\end{tabular}


Tabla 5. Valores de Capacidad admisible para la Calicata C-5

\begin{tabular}{|c|c|c|c|}
\hline Df $(\mathbf{m})$ & $\mathbf{B}(\mathbf{m})$ & $\begin{array}{c}\mathbf{Q}(\mathbf{u} \mathbf{l t}) \\
\mathbf{T n} / \mathbf{m} 2\end{array}$ & $\begin{array}{c}\mathbf{Q}(\mathbf{a d m}) \\
\mathbf{K g} . \mathbf{c m} \mathbf{2}\end{array}$ \\
\hline 0.60 & 0.80 & 11.04 & 0.37 \\
\hline 0.60 & 1.00 & 11.83 & 0.39 \\
\hline 0.60 & 1.20 & 12.62 & 0.42 \\
\hline 0.60 & 1.40 & 13.41 & 0.45 \\
\hline 0.60 & 1.60 & 14.21 & 0.47 \\
\hline 0.80 & 0.80 & 13.67 & 0.46 \\
\hline 0.80 & 1.00 & 14.46 & 0.48 \\
\hline 0.80 & 1.20 & 15.25 & 0.51 \\
\hline 0.80 & 1.40 & 16.04 & 0.53 \\
\hline 0.80 & 1.60 & 16.83 & 0.56 \\
\hline 1.00 & 0.80 & 16.30 & 0.54 \\
\hline 1.00 & 1.00 & 17.09 & 0.57 \\
\hline 1.00 & 1.20 & 17.88 & 0.60 \\
\hline 1.00 & 1.40 & 18.67 & 0.62 \\
\hline 1.00 & 1.60 & 19.46 & 0.65 \\
\hline 1.20 & 0.80 & 18.92 & 0.63 \\
\hline 1.20 & 1.00 & 19.71 & 0.66 \\
\hline 1.20 & 1.20 & 20.51 & 0.68 \\
\hline 1.20 & 1.40 & 21.30 & 0.71 \\
\hline 1.20 & 1.60 & 22.09 & 0.74 \\
\hline & & & \\
\hline
\end{tabular}

Tabla 6. Valores de Capacidad admisible para la Calicata C-6

\begin{tabular}{|c|c|c|c|c|c|c|c|}
\hline Df $(m)$ & $B(m)$ & $\begin{array}{c}\text { Q(ult) } \\
\text { Tn/m2 }\end{array}$ & $\begin{array}{l}\mathrm{Q} \text { (adm) } \\
\mathrm{Kg} . / \mathrm{cm} 2 \\
\end{array}$ & Df $(m)$ & $B(m)$ & $\begin{array}{c}\text { Q(ult) } \\
\text { Tn/m2 }\end{array}$ & $\begin{array}{l}\mathrm{Q}(\mathrm{adm}) \\
\mathrm{Kg} \cdot / \mathrm{cm} 2 \\
\end{array}$ \\
\hline 0.60 & 0.80 & 11.66 & 0.39 & 1.50 & 0.80 & 24.10 & 0.80 \\
\hline 0.60 & 1.00 & 12.49 & 0.42 & 1.50 & 1.00 & 24.94 & 0.83 \\
\hline 0.60 & 1.20 & 13.33 & 0.44 & 1.50 & 1.20 & 25.78 & 0.86 \\
\hline 0.60 & 1.40 & 14.17 & 0.47 & 1.50 & 1.40 & 26.62 & 0.89 \\
\hline 0.60 & 1.60 & 15.01 & 0.50 & 1.50 & 1.60 & 27.46 & 0.92 \\
\hline 0.80 & 0.80 & 14.42 & 0.48 & 2.00 & 0.80 & 31.02 & 1.03 \\
\hline 0.80 & 1.00 & 15.26 & 0.51 & 2.00 & 1.00 & 31.86 & 1.06 \\
\hline 0.80 & 1.20 & 16.10 & 0.54 & 2.00 & 1.20 & 32.70 & 1.09 \\
\hline 0.80 & 1.40 & 16.94 & 0.56 & 2.00 & 1.40 & 33.54 & 1.12 \\
\hline 0.80 & 1.60 & 17.78 & 0.59 & 2.00 & 1.60 & 34.38 & 1.15 \\
\hline 1.00 & 0.80 & 17.19 & 0.57 & 2.50 & 0.80 & 37.94 & 1.26 \\
\hline 1.00 & 1.00 & 18.03 & 0.60 & 2.50 & 1.00 & 38.78 & 1.29 \\
\hline 1.00 & 1.20 & 18.87 & 0.63 & 2.50 & 1.20 & 39.62 & 1.32 \\
\hline 1.00 & 1.40 & 19.71 & 0.66 & 2.50 & 1.40 & 40.45 & 1.35 \\
\hline 1.00 & 1.60 & 20.54 & 0.68 & 2.50 & 1.60 & 41.29 & 1.38 \\
\hline 1.20 & 0.80 & 19.96 & 0.67 & & & & \\
\hline 1.20 & 1.00 & 20.79 & 0.69 & & & & \\
\hline 1.20 & 1.20 & 21.63 & 0.72 & & & & \\
\hline 1.20 & 1.40 & 22.47 & 0.75 & & & & \\
\hline 1.20 & 1.60 & 23.31 & 0.78 & & & & \\
\hline
\end{tabular}


Tabla 7. Valores de Capacidad admisible para la Calicata C-7

\begin{tabular}{|c|c|c|c|}
\hline Df (m) & $\mathbf{B}(\mathbf{m})$ & $\begin{array}{c}\mathbf{Q}(\mathbf{u l t}) \\
\mathbf{T n} / \mathbf{m} 2\end{array}$ & $\begin{array}{c}\mathbf{Q}(\mathbf{a d m}) \\
\mathbf{K g} . / \mathbf{c m} 2\end{array}$ \\
\hline 0.60 & 0.80 & 12.47 & 0.42 \\
\hline 0.60 & 1.00 & 13.35 & 0.44 \\
\hline 0.60 & 1.20 & 14.22 & 0.47 \\
\hline 0.60 & 1.40 & 15.10 & 0.50 \\
\hline 0.60 & 1.60 & 15.98 & 0.53 \\
\hline 0.80 & 0.80 & 15.45 & 0.52 \\
\hline 0.80 & 1.00 & 16.33 & 0.54 \\
\hline 0.80 & 1.20 & 17.21 & 0.57 \\
\hline 0.80 & 1.40 & 18.09 & 0.60 \\
\hline 0.80 & 1.60 & 18.96 & 0.63 \\
\hline 1.00 & 0.80 & 18.44 & 0.61 \\
\hline 1.00 & 1.00 & 19.32 & 0.64 \\
\hline 1.00 & 1.20 & 20.19 & 0.67 \\
\hline 1.00 & 1.40 & 21.07 & 0.70 \\
\hline 1.00 & 1.60 & 21.95 & 0.73 \\
\hline
\end{tabular}

Tabla 8. Valores de Capacidad admisible para la Calicata C-8

\begin{tabular}{|c|c|c|c|}
\hline Df (m) & $\mathbf{B}(\mathbf{m})$ & $\begin{array}{c}\mathbf{Q}(\mathbf{u l t}) \\
\mathbf{T n} / \mathbf{m} 2\end{array}$ & $\begin{array}{c}\mathbf{Q}(\mathbf{a d m}) \\
\mathbf{K g} . \mathbf{c m} 2\end{array}$ \\
\hline 0.60 & 0.80 & 8.50 & 0.28 \\
\hline 0.60 & 1.00 & 9.06 & 0.30 \\
\hline 0.60 & 1.20 & 9.61 & 0.32 \\
\hline 0.60 & 1.40 & 10.16 & 0.34 \\
\hline 0.60 & 1.60 & 10.72 & 0.36 \\
\hline 0.80 & 0.80 & 10.60 & 0.35 \\
\hline 0.80 & 1.00 & 11.15 & 0.37 \\
\hline 0.80 & 1.20 & 11.71 & 0.39 \\
\hline 0.80 & 1.40 & 12.26 & 0.41 \\
\hline 0.80 & 1.60 & 12.81 & 0.43 \\
\hline 1.00 & 0.80 & 12.69 & 0.42 \\
\hline 1.00 & 1.00 & 13.25 & 0.44 \\
\hline 1.00 & 1.20 & 13.80 & 0.46 \\
\hline 1.00 & 1.40 & 14.36 & 0.48 \\
\hline 1.00 & 1.60 & 14.91 & 0.50 \\
\hline
\end{tabular}

Se propone generar mapas de isovalores de capacidad admisible del Sector Siete del distrito Alto Alianza o Alto Tacna, entre los km 6+000 al km 7+000 de la carretera Tacna Tarata.

Los mapas de isovalores de la capacidad portante, corresponde a profundidad de desplante de 0.8 y $1.20 \mathrm{~m}$, para guardar consistencia con las viviendas de dos a tres pisos que tienen estos desplantes en sus cimientos. Los resultados de capacidad admisible están referidos a profundidades entre $0.80 \mathrm{~m}$ a $2.5 \mathrm{~m}$, sin analizar los afloramientos rocosos, dado que las rocas tienen parámetros de resistencia distintos y elevados. Para las exploraciones C-1; C-4 y C-6 se analizó profundidades mayores a $1.20 \mathrm{~m}$ y para las C-2; C-3; C-5; C7 y C8 profundidades menores de $1.20 \mathrm{~m}$. Se ha considerado que toda el área presenta adecuada capacidad portante para la demanda de cargas de las edificaciones. Para profundidades de $0.80 \mathrm{~m}$ y $1.20 \mathrm{~m}$ se obtuvieron los resultados siguientes:

Tabla 9. Valores de qadm para $\mathrm{Df}=0.80 \mathrm{~m}$ y $1.20 \mathrm{~m}$.

\begin{tabular}{|c|c|c|c|c|}
\hline \multirow{3}{*}{ Calicata } & \multirow{3}{*}{ Ubicación } & \multirow{3}{*}{$\begin{array}{c}\text { Prof. } \\
\text { calicata } \\
\text { (m) }\end{array}$} & \multirow{2}{*}{\multicolumn{2}{|c|}{$\frac{\text { qadm }}{\mathrm{Df}(\mathrm{m})}$}} \\
\hline & & & & \\
\hline & & & 0.80 & 1.20 \\
\hline C-1 & $\begin{array}{l}\text { Asociación criadores de animales menores } \\
\text { Alto Tacna }\end{array}$ & 2.50 & 0.6 & 0.81 \\
\hline $\mathrm{C}-2$ & $\begin{array}{l}\text { Asociación criadores de porcinos Virgen de } \\
\text { Chapi }\end{array}$ & 0.80 & 0.61 & \\
\hline$C-3$ & $\begin{array}{l}\text { Asociación criadores de animales menores } \\
\text { Las Pascuas }\end{array}$ & 1.10 & 0.39 & 0.54 \\
\hline C-4 & Asociación de granjas Nueva Tarata & 2.00 & 0.5 & 0.68 \\
\hline C-5 & $\begin{array}{l}\text { Asociación Artesanal Taller Vivienda Héroes } \\
\text { del Alto de la Alianza }\end{array}$ & 1.20 & 0.48 & \\
\hline C-6 & $\begin{array}{l}\text { Asociación Hermandad de la Santísima Cruz } \\
\text { del cerro intiorko }\end{array}$ & 2.20 & 0.51 & 0.69 \\
\hline C-7 & $\begin{array}{l}\text { Asociación criadores de aves Alto Santa Cruz } \\
\text { Cerro intiorko }\end{array}$ & 0.90 & 0.54 & \\
\hline C.8 & $\begin{array}{l}\text { Asociación criadores de animales menores } \\
\text { Las Vegas }\end{array}$ & 0.80 & 0.37 & \\
\hline
\end{tabular}


Con los valores de la tabla anterior se muestra el mapa de isovalores de capacidad admisible para una profundidad de desplante a $1.20 \mathrm{~m}$. Se observa zona de afloramiento rocoso y zona de suelo de capacidad entre 0.54 a $0.81 \mathrm{~kg} / \mathrm{cm}^{2}$.

Los resultados de isovalores de la capacidad de carga admisible de los suelos en la zona de investigación, se observa que presentan una capacidad portante baja, que son propios de suelos de arena limosa de procedencia volcánica. De los perfiles se desprende que los suelos que se encuentran al lado izquierdo de la vía son la que tienen mayor profundidad de estrato que las que están al lado derecho, por lo que se plantea el uso de cimentaciones aisladas en el lado derecho de la vía y cimentaciones conectadas mediante vigas y/o plateas de cimentación en el lado izquierdo de la vía Tacna - Tarata.

\section{DISCUSIÓN}

Las propiedades físicas del suelo y de resistencia caracterizan al suelo de la zona de estudio, a una profundidad de desplante de 0.8 y $1.20 \mathrm{~m}$ como un suelo arena limosa (SM) no plástica, con una densidad in situ es de $1.55 \mathrm{gr} / \mathrm{cc}$ a 1.87 , con una coloración de rosado a claro, cohesión cero y ángulo de fricción del suelo que varía de $25.2^{\circ}$ a $31.5^{\circ}$, confirmando un material propio de cenizas volcánicas. Por debajo de esta capa existe un afloramiento rocoso de la formación huaylillas con una profundidad indefinida. Los resultados de la capacidad de carga admisible para una profundidad de desplante de $1.20 \mathrm{~m}$ y para un ancho de cimentación cuadrada de $1.0 \mathrm{~m}$ son los siguientes: de $0.81 \mathrm{~kg} / \mathrm{cm}^{2}$ en la calicata C-1; 0.54 $\mathrm{kg} / \mathrm{cm}^{2}$ en la calicata C-3, $0.56 \mathrm{~kg} / \mathrm{cm}^{2}$ en la calicata C-4 y $0.69 \mathrm{~kg} / \mathrm{cm}^{2}$ en la calicata C-6, en otras calicatas se presenta afloramientos rocosos. Los valores nos indican capacidades de carga admisible similares a los resultados del estudio de INDECI para cimentaciones superficiales. Se han elaborado mapas de isovalores de la capacidad de carga en el Sector Siete del distrito de Alto de la Alianza, determinando la Capacidad Admisible del suelo para una profundidad de $0.80 \mathrm{~m}$ es de $0.37 \mathrm{~kg} / \mathrm{cm} 2$ a $0.61 \mathrm{~kg} / \mathrm{cm} 2$ y para una profundidad de $1.20 \mathrm{~m}$ es de $0.54 \mathrm{~kg} / \mathrm{cm} 2$ a $0.81 \mathrm{~kg} / \mathrm{cm} 2$. Con un fondo rocoso variable en su profundidad. Con los mapas de isovalores se fortalece el actuar de las autoridades al tomar decisiones frente al crecimiento urbano indiscriminado y a mitigar los daños por sismos, definiendo áreas para el uso de cimentaciones aisladas y zonas con cimentaciones conectadas y/o plateas de cimentación, con relación a los suelos del área de investigación que está definida como arenas limosos. Se recomienda a la Municipalidad Distrital del Alto de la Alianza, tomar en consideración los resultados de las propiedades físicas, los valores de resistencia de los suelos y de los valores de la capacidad portante de los suelos de esta tesis para ser considerados en la planificación de la expansión urbana del distrito. Asimismo, se recomienda a la Municipalidad Provincial de Tacna, realizar ensayos de exploración indirecta o no destructivos mediante métodos de exploración geofísica sismológica (reflexión sísmica y refracción sísmica). A fin de determinar las características físicas y dinámicas del suelo a mayores profundidades, para ampliar el sustento técnico de la zonificación de las expansiones urbanas con fines de vivienda. Se recomienda a los posesionarios de terrenos de la zona de estudio el uso de cementos resistentes al ataque de sulfatos (sales) en el proceso constructivo de la subestructura (cimentación), siguiendo los lineamientos del estudio de mecánica de suelos para su construcción.

\section{REFERENCIAS BIBLIOGRAFICAS}

Aguilar, Morales, Chunga, Evelin \& Del Castillo (1998). Acelerogramas y espectros inferidos del terremoto 1998 (Mw 7,2). Alva, J. (2012). Diseño de Cimentaciones, Lima Perú

Atala, César (2011) Estudio experimental sobre correlaciones en suelos granulares finos (arenas) compactados, usando equipos de penetración. Tesis de Post Grado (maestro en ciencias) UNIPeru. 
Aya, J (2015) Estudio de suelos con fines de cimentación en el cerro Intiorko -distrito Alto de la Alianza región Tacna. Ponencia en XIX Congreso nacional de Ingeniería Civil - Huaraz. Perú.

Bonett D., Ricardo L., (2003), Tesis Doctoral "Vulnerabilidad y riesgo sísmico de edificios. Aplicación a entornos urbanos en zonas de amenaza alta y moderada, Universidad Politécnica de Cataluña. Escuela Técnica Superior de Ingenieros de Caminos, Barcelona, España.

Calumani Aguilar, (2009) Estudio de Suelos para Cimentaciones en edificaciones de la expansión urbana del distrito de Ciudad Nueva - Tacna. Tesis pre grado UPT.

Chipana, L. (2014) Geología urbana y procesos de zonificación territorial en la ciudad de Tacna. Tesis pregrado UNA

Chura, Edgar (2012) Estudio de Riesgo Sísmico en el Distrito de Ciudad Nueva - Tacna. Ciencia y desarrollo.

Crespo Villalaz, C. (2005) Mecánica de suelos y cimentaciones, 5ạ Edición; México: Editorial LIMUSA.

Delgado Vargas M. (2000) Ingeniería de Cimentaciones, Fundamentos e Introducción al Análisis Geotécnico.

Eulalio Juárez Badillo y Alfonso Rico Rodríguez. (2003) Mecánica de suelos, tomo II. 2a

Fletcher, Gordon A. (2002) Estudios de Suelos y Cimentaciones en la Industria de la Construcción

Huanacuni Quispe, Diana. (2015) Caracterización Dinámica de los suelos en la ciudad de Tacna. Tesis de pregrado de la UPT

INDECI, (2007). Programa de prevención y medidas de mitigación ante desastres de la ciudad de Tacna.

Lee, Ian K. y otros. (1983) Geotechnical engineering. 1a Edición; Estados Unidos: Editorial Pitman.

Municipalidad Provincial de Tacna, (2015) Plan d Desarrollo urbano de la Ciudad de Tacna 2015-2025, Tacna.

Lambert, W. y Whitman, R. (1991) Mecánica de suelos. 1ạ Edición; México.

Reglamento Nacional de Edificaciones NTE 030 (2006) Diseño Sismo Resistente, Lima Perú

Safina M., Salvador, (2002), "Vulnerabilidad sísmica de edificaciones esenciales análisis de su contribución al riesgo sísmico, Tesis Doctoral, Universidad Politécnica de Cataluña, Escuela Técnica Superior de Ingenieros de Caminos, Barcelona, España.

Silva Aranibar, Juan. (2004) Estudio de Suelos para Cimentaciones del Cono Norte de la Ciudad de Tacna. Tesis de pre grado UPT-Tacna

Terzaghi, Karl. (1949), Mecánica teórica de suelos. 2ª Edición; Buenos Aires

Peck, Ralph B. y otros. (1996), Ingeniería de cimentaciones.

Vásquez Quispe, Ada. (2012) Estudio de Suelos para Cimentaciones en edificaciones de la expansión urbana del distrito de G. Albarracín L - Tacna. Tesis pre grado UPT.

Vera, Luis (1990) Microzonificación sísmica de la ciudad de Tacna, Investigación UNESCO.

Whitlow, Roy. (2000), Fundamentos de mecánica de suelos.

Zegarra, J. (2000) Mecánica de Suelos. PUCP - Lima. Perú 